\title{
CONCENTRACIÓN NUTRIMENTAL DE BIOCARBÓN DE CASCARILLA DE ARROZ
}

\section{NUTRIENT CONCENTRATION OF RICE HUSK BIOCHAR}

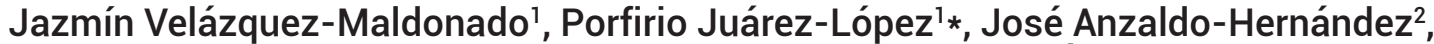 \\ Gelacio Alejo-Santiago ${ }^{3}$, Luis Alonso Valdez-Aguilar ${ }^{4}$, Irán Alia-Tejacal ${ }^{1}$, \\ Víctor López-Martínez', G. Alicia Pérez-Arias' y Dagoberto Guillén-Sánchez ${ }^{1}$
}

\begin{abstract}
'Universidad Autónoma del Estado de Morelos, Facultad de Ciencias Agropecuarias, Cuernavaca, Morelos, México. ${ }^{2}$ Universidad de Guadalajara, Centro Universitario de Ciencias Exactas e Ingenierías, Guadalajara, Jalisco, México. ${ }^{3}$ Universidad Autónoma de Nayarit, Unidad Académica de Agricultura, Xalisco, Nayarit, México. ${ }^{4}$ Universidad Autónoma Agraria Antonio Narro, Departamento de Horticultura, Saltillo, Coahuila México.
\end{abstract}

*Autor para correspondencia (porfiriojlopez@yahoo.com)

\section{RESUMEN}

El biocarbón es un material sólido poroso que se produce por la conversión termoquímica de materiales orgánicos en un ambiente limitado o en ausencia de oxígeno; tiene propiedades físico-químicas aptas para el almacenamiento a largo plazo de carbono y, potencialmente, mejora la fertilidad de los suelos. El objetivo del presente estudio fue evaluar la concentración nutrimental en biocarbón elaborado con cascarilla de arroz (Oryza sativa L.) y adicionado con ácido cítrico, maleico y propiónico al 5 y $10 \%$ como catalizadores. El biocarbón se elaboró con la técnica de carbonización hidrotérmica a $200{ }^{\circ} \mathrm{C}$ durante $19 \mathrm{~h}$. Se determinó el rendimiento de biocarbón, concentración de materia orgánica y de N, P, K, Ca, Mg, Cu, Fe, Mn, Zn, Si y Na. Los mayores rendimientos de biocarbón (66 \%) se obtuvieron cuando se añadieron los ácidos maleico y cítrico al $10 \%$. El mayor contenido de materia orgánica (71 $\%$ ) se obtuvo con ácido maleico al $5 \%$. En general, los tres macronutrimentos con mayor concentración $\left(\mathrm{mg} \mathrm{kg}^{-1}\right)$ fueron Ca $(4386-10,988)$, N (30674467) y K (327-3530), mientras que los micronutrimentos con mayor concentración ( $\left.\mathrm{mg} \mathrm{kg}^{-1}\right)$ fueron Fe (77.1-238.3) y Mn (75.4-281.4), así como el elemento benéfico $\mathrm{Na}$ (92.8-160.0). Por sus características de rendimiento, concentraciones de materia orgánica y nutrimental, el biocarbón de cascarilla de arroz potencialmente se podría usar como mejorador de suelos agrícolas.

Palabras clave: Oryza sativa, carbonización hidrotérmica, biomasa, macronutrimentos, micronutrimentos.

\section{SUMMARY}

Biochar is a porous, solid material that is produced by the thermochemical conversion of organic materials under a limited or oxygen-free environment; it has physical and chemical properties suitable for the long-term storage of carbon, and potentially, improves the fertility of soils. The objective of this study was to evaluate the nutrient concentration of biochar prepared with rice (Oryza sativa L.) husk and added with citric, maleic and propionic acids at 5 and $10 \%$ as catalysts. The biochar was prepared through the hydrothermal carbonization technique at $200{ }^{\circ} \mathrm{C}$ for $19 \mathrm{~h}$. The yield of biochar and concentration of organic matter, $\mathrm{N}, \mathrm{P}, \mathrm{K}, \mathrm{Ca}, \mathrm{Mg}, \mathrm{Cu}, \mathrm{Fe}, \mathrm{Mn}, \mathrm{Zn}$, Si and $\mathrm{Na}$ were determined. The highest yields of biochar $(66 \%)$ were obtained when $10 \%$ maleic and citric acids were added. The highest content of organic matter $(71 \%)$ was obtained with $5 \%$ maleic acid. In general, the three macronutrients with the highest concentration ( $\mathrm{mg} \mathrm{kg}^{-1}$ ) were $\mathrm{Ca}(4386-10,988), \mathrm{N}(3067-4467)$ and $\mathrm{K}$ (327-3530), while the micronutrients with the highest concentration $\left(\mathrm{mg} \mathrm{kg}^{-1}\right)$ were Fe (77.1-238.3) and $\mathrm{Mn}$ (75.4-281.4), as well as the beneficial element $\mathrm{Na}$ (92.8-160.0). Due to its yield, organic matter and nutrient concentrations, the rice husk biochar could be used, potentially, as an improver of agricultural soils.
Index words: Oryza sativa, hydrothermal carbonization, biomass, macronutrients, micronutrients.

\section{INTRODUCCIÓN}

El uso intensivo de los suelos ha ocasionado que éstos se degraden y que pierdan su fertilidad, por lo que se han empleado técnicas agronómicas para su mejoramiento Entre las prácticas agrícolas que se realizan para mejorar la fertilidad del suelo se encuentra la incorporación de compostas, residuos de cosechas, pajas, cascarillas y recientemente, el uso de biocarbón (Adeyemi e Idowu, 2017; Escalante et al., 2016; Medina y Medina, 2018).

El biocarbón es un material sólido y poroso que se produce por la conversión termoquímica de materiales orgánicos en un ambiente limitado o carente de oxígeno, el cual tiene propiedades físico-químicas aptas para el almacenamiento a largo plazo de carbonoy, potencialmente, la mejora de la fertilidad de los suelos (Ibarrola et al., 2013). El biocarbón es el resultado de la carbonización de materias primas vegetales, como residuos de cosecha, plantas secas, biomasa de árboles, desechos de papel cascarilla de arroz y desechos de varias especies vegetales (Escalante et al., 2016). Se puede obtener biocarbón de casi cualquier biomasa, pero es particularmente apropiada la de origen vegetal, sobre todo la constituida por materiales lignocelulósicos, que son el constituyente predominante de la vegetación terrestre, después del agua (QuesadaKimsey, 2012).

Existen varias tecnologías termoquímicas para producir biocarbón, tales como la pirólisis (lenta, rápida y ultrarrápida), gasificación y conversión hidrotérmica (HTC) (Zheng et al., 2016). Esta última técnica consiste en someter el material a temperaturas altas, sumergido en agua, sin permitir que haya ebullición. Se ha observado que con este tipo de reacción es posible carbonizar materiales 
lignocelulósicos sólidos y se obtienen como productos materiales carbonosos nanoestructurados (Baccile et al., 2010).

Entre los factores que afectan el mecanismo y la cinética de las reacciones de pirolisis en la producción de biocarbón, se encuentran la composición del sustrato (material vegetal), intervalo de calentamiento, temperatura y presión del biorreactor, la atmósfera ambiental presente y el uso de catalizadores. Estas variables tienen un efecto profundo en la secuencia y cinética de las reacciones termoquímicas $y$, por tanto, en el rendimiento de los productos formados (Escalante et al., 2016; Kambo y Dutta, 2015). Al respecto del uso de catalizadores, Lynam et al. (2012) y Lynam et al. (2011) sugieren el uso de ácidos o sales como catalizadores durante el proceso de HTC, ya que favorecen la eficiencia de hidrólisis de celulosa y hemicelulosa, lo que puede influir en el rendimiento del biocarbón producido; además, el uso de catalizadores ácidos puede influir en la concentración nutrimental del biocarbón (Centurion et al., 2017).

Existen estudios que indican que la aplicación de biocarbón en suelos agrícolas mejora el rendimiento en diversos cultivos como Amaranthus viridis e Ipomoea reptans (Rosenani et al., 2014), Solanum lycopersicum (Villamagua et al., 2014) e Ipomoea aquatica (Varela et al., 2013); además, también mejoran las propiedades físicas y químicas del suelo (Ding et al., 2016; Schulz et al., 2013). Adicionalmente, el biocarbón funciona como agente secuestrante del carbono presente en la atmósfera, lo que puede contribuir a la mitigación del cambio climático (Adeyemi e Idowu, 2017; Gurwick et al., 2013).

En México, en el año 2017 se cosecharon 41,560 ha de arroz, con una producción de 265,567 t (SIAP, 2019). Si se considera que el peso de la cascarilla en arroz seco es de aproximadamente 27 \% (Rodríguez y Grant, 2015), se estima que en ese mismo año se produjeron $71,703 \mathrm{t}$ de cascarilla, lo que significa que potencialmente se tiene la materia prima para producir biocarbón de cascarilla de arroz y, al mismo tiempo, aprovechar el subproducto de este cultivo.

Por otro lado, la mayoría de los estudios de caracterización química de biocarbón se enfocan en la concentración de C, H, O y N (Kambo y Dutta, 2015; Schneider et al., 2011) y son escasos los reportes que incluyen el contenido nutrimental de otros macronutrimentos de importancia en la nutrición de cultivos, y más raros aun los que abordan la concentración de micronutrimentos. Por lo anterior, el objetivo del presente estudio fue evaluar la concentración nutrimental de biocarbón elaborado a partir de cascarilla de arroz, adicionado con los ácidos cítrico, maleico y propiónico como catalizadores.

\section{MATERIALES Y MÉTODOS}

\section{Elaboración del biocarbón y tratamientos}

Se utilizó cascarilla de arroz proveniente de un molino comercialubicado en Cuautla, Morelos, México. El biocarbón se obtuvo por medio de la técnica de carbonización hidrotérmica (HTC) con la metodología reportada por Reza et al. (2015) con algunas modificaciones. Se pesaron $25 \mathrm{~g}$ de cascarilla de arroz (base seca), con humedad inicial de $10.9 \%$; después, se adicionó agua a las muestras hasta obtener una humedad final de $50 \%$. Posteriormente, a cada muestra se le adicionó el catalizador correspondiente (ácido cítrico, ácido maleico o ácido propiónico) en concentraciones de 5 y $10 \%$ con respecto al peso de la cascarilla en base seca. Como testigo se utilizó la misma mezcla sin adicionar ácidos. Posteriormente, las muestras se colocaron en un reactor (modelo JAYME Deutsch and Neumann ${ }^{\circledR}$, Berlín, Alemania) durante $19 \mathrm{~h}$ a $200{ }^{\circ} \mathrm{C}$; después, se dejó enfriar el reactor a temperatura ambiente. Al material obtenido se le realizó un triple lavado con agua destilada-etanol 96 \%-agua destilada. Finalmente, el biocarbón se secó en una estufa de aire circulante (modelo F210 Felisa ${ }^{\circledR}$, Guadalajara, Jalisco, México) a 105 ${ }^{\circ} \mathrm{C}$ durante $8 \mathrm{~h}$.

\section{Rendimiento de biocarbón}

El rendimiento del biocarbón se determinó mediante la diferencia entre el peso de la cascarilla cruda (biomasa inicial) y el peso de la cascarilla convertida en biocarbón; posteriormente, el valor obtenido se expresó en términos de porcentaje.

\section{Materia orgánica}

La concentración de materia orgánica del biocarbón se determinó por calcinación a $550{ }^{\circ} \mathrm{C}$ durante 5 min (Wang y Mulligan, 2009), en una mufla (12-PR-300, Hobersal ${ }^{\circledR}$, Barcelona, España). Se expresó en porcentaje tras considerar el peso original del biocarbón y el peso del biocarbón después del proceso de incineración.

\section{Concentración nutrimental}

Se realizó una extracción en digestión húmeda mediante los procedimientos descritos por Alcántar y Sandoval (1999). Para el caso del N se utilizó una mezcla de ácido sulfúrico con ácido salicílico, mientras que para el $\mathrm{Ca}, \mathrm{Mg}$, $\mathrm{P}, \mathrm{K}$, Si y Na se usó una mezcla con ácido nítrico y ácido perclórico. Se determinó el contenido total de $\mathrm{N}$ por el método semi-microkjeldahl, $\mathrm{P}$ por colorimetría, con un 
espectrofotómetro (Hach $\AA$, Loveland, Colorado, EUA); $\mathrm{K}$ y $\mathrm{Na}$ por flamometría con un flamómetro (Flame Photometer 410®, Hamburgo, Alemania); Ca, Mg y Si por espectrofotometría con un espectrofotómetro de absorción atómica (Varian SpectrAA®, Melbourne, Australia).

\section{Estructura del biocarbón}

Se molieron $0.01 \mathrm{~g}$ de biocarbón y la muestra se fijó en un portamuestras con una cinta adhesiva de doble cara, cuidando no dejar material carbonoso sin adherir. Las muestras se recubrieron mediante pulverización con oro (Varela et al., 2013). Se analizó con un microscopio electrónico de barrido (JEOL 6400®, Houghton, Michigan, EUA).

\section{Diseño experimental y análisis estadístico}

El diseño experimental fue completamente al azar con cinco repeticiones. A los resultados se les realizó análisis de varianza. Cuando el análisis de varianza detectó diferencia estadística, se aplicó una prueba de medias de Tukey $(P \leq 0.05)$ mediante el programa estadístico SAS versión 9.1 (SAS Institute, 2004).

\section{RESULTADOS Y DISCUSIÓN}

\section{Rendimiento del biocarbón}

Hubo diferencias $(P \leq 0.05)$ en el rendimiento de biocarbón en función de los catalizadores empleados (Cuadro 1), el cual varió de 54 \% (ácido propiónico $5 \%$ ) hasta $66 \%$ (ácido cítrico $10 \%$ y ácido maleico $10 \%$ ). No se encontraron reportes en la literatura revisada con relación al rendimiento de biocarbón asociado con la adición de los ácidos empleados en el presente estudio; sin embargo, Centurion et al. (2017), en la elaboración de biocarbón a partir de mezclas de vinaza y bagazo de caña de azúcar (Saccharum officinarum), reportaron que al aplicar ácido bórico a concentración de 0.1 M obtuvieron 20 \% de rendimiento, mientras que al aplicar ácido sulfúrico en la misma concentración el rendimiento fue de $35 \%$.

En general, los resultados son aproximados a los reportados por Kalderis et al. (2014), quienes al elaborar biocarbón de cascarilla de arroz reportaron rendimientos de entre 58 y $66 \%$. Por otro lado, los resultados obtenidos son superiores a los de Alburquerque et al. (2014), quienes reportaron rendimiento de 37.8 \% en biocarbón elaborado a partir de paja de trigo (Triticum spp.), a los de Onay (2014), quien encontró rendimiento de 18 \% en biocarbón de semillas de laurel (Laurus nobilis L.) y a los de Medina y Medina (2018), quienes en biocarbón de residuos de aguacate (Persea americana) reportaron un rendimiento de $16 \%$. Al respecto, el rendimiento del biocarbón depende del tipo de material vegetal empleado, intervalo de calentamiento, temperatura y presión del biorreactor y el uso de catalizadores (Kambo y Dutta, 2015). Asimismo, la adición de catalizadores ácidos puede influir en el rendimiento, debido a que favorecen la degradación de la celulosa y hemicelulosa (Lynam et al., 2011; 2012).

\section{Materia orgánica}

Se observaron diferencias $(P \leq 0.05)$ en la concentración de materia orgánica del biocarbón con los catalizadores utilizados, la cual varió de 65 a 71 \% (Cuadro 1). Centurion et al. (2017) reportaron diferencias en la concentración de

Cuadro 1. Rendimiento, materia orgánica (MO) y concentración de macronutrimentos de biocarbón elaborado de cascarilla de arroz con tres catalizadores.

\begin{tabular}{|c|c|c|c|c|c|c|c|}
\hline \multirow{2}{*}{ Tratamiento } & Rendimiento & $\mathrm{MO}$ & $\mathrm{N}$ & $\mathrm{P}$ & K & $\mathrm{Ca}$ & $\mathrm{Mg}$ \\
\hline & \multicolumn{2}{|c|}{$(\%)$} & \multicolumn{5}{|c|}{$\left(\mathrm{mg} \mathrm{kg}^{-1}\right)$} \\
\hline Agua (Testigo) & $50 f$ & $66 c$ & $3067 \mathrm{c}$ & $164 \mathrm{~g}$ & $406 f$ & $4387 d$ & $483 \mathrm{~g}$ \\
\hline Ácido maleico 5 \% & $64 \mathrm{~b}$ & $71 \mathrm{a}$ & $3367 \mathrm{c}$ & $475 d$ & 979 c & $8788 b$ & $804 \mathrm{e}$ \\
\hline Ácido maleico 10 \% & $66 a$ & $65 c$ & $3133 c$ & $345 e$ & $327 \mathrm{~g}$ & $6587 c$ & $554 f$ \\
\hline Ácido cítrico 5 \% & $61 c$ & $68 b$ & $3700 \mathrm{bc}$ & $215 f$ & $722 \mathrm{~d}$ & $4386 d$ & $911 d$ \\
\hline Ácido cítrico 10 \% & $66 a$ & $70 a b$ & 3267 c & $504 c$ & $1,117 b$ & 10988 a & $983 \mathrm{c}$ \\
\hline Ácido propiónico 5 \% & $54 \mathrm{e}$ & $66 c$ & $4233 a b$ & $527 \mathrm{~b}$ & $544 \mathrm{e}$ & $10978 a$ & $1018 b$ \\
\hline Ácido propiónico 10 \% & $58 d$ & $66 c$ & 4467 a & $849 a$ & 3530 a & $5487 d$ & $1447 a$ \\
\hline CV $(\%)$ & 1.7 & 1.0 & 6.8 & 9.3 & 11.1 & 12.8 & 10.4 \\
\hline DSH (0.05) & 1.1 & 2.1 & 699.5 & 261.2 & 35.9 & $1,947.3$ & 34.7 \\
\hline
\end{tabular}

Medias con letras iguales dentro de las columnas no son estadísticamente diferentes (Tukey, P $\leq 0.05)$, CV: coeficiente de variación, DSH: diferencia significativa honesta. 
materia orgánica al adicionar los ácidos sulfúrico, fosfórico y bórico a concentración de $0.1 \mathrm{M}$ en la elaboración de biocarbón a partir de mezclas de vinaza y bagazo de caña de azúcar. Independientemente del catalizador empleado, los valores obtenidos son ligeramente superiores a los de George et al. (2017), quienes reportaron 60.9 \% de materia orgánica en biocarbón de residuos de poda de olivo (Olea europaea); asimismo, los resultados son superiores a los reportados por Medina y Medina (2018), quienes reportaron de 18.8 a 22.6 \% de materia orgánica en biocarbón de caña de azúcar y de $59.2 \%$ en biocarbón de cachaza de caña. Al igual que el rendimiento, la materia orgánica depende del material vegetal utilizado para elaborar los biocarbones.

\section{Concentración de macronutrimentos}

Hubo diferencias $(P \leq 0.05)$ en la concentración de macronutrientes en función del catalizador empleado en la elaboración del biocarbón (Cuadro 1). En este sentido, las concentraciones mayores de N, P, K y Mg se obtuvieron con ácido propiónico $10 \%$ y para el Ca con ácido cítrico $10 \%$ y ácido propiónico $5 \%$. Lo anterior concuerda con lo reportado por Centurion et al. (2017), quienes encontraron diferencias en la concentración de macronutrimentos (C, $\mathrm{H}, \mathrm{N}, \mathrm{S}$ y O) al adicionar los ácidos sulfúrico, fosfórico y bórico a concentración de $0.1 \mathrm{M}$ en la elaboración de biocarbón con mezclas de vinaza y bagazo de caña de azúcar. Los resultados del presente estudio indican que la adición de catalizadores favoreció la concentración de macronutrimentos en biocarbón de cascarilla de arroz, ya que los valores fueron superiores a los del testigo.
En general, independientemente del catalizador empleado, la concentración de macronutrimentos en orden decreciente fue como sigue: $\mathrm{Ca}>\mathrm{N}>\mathrm{Mg}>\mathrm{K}>\mathrm{P}$ (Cuadro 1). Esta secuencia de concentraciones es diferente a la reportada por George et al. (2017), quienes en biocarbón de maíz (Zea mays) ensilado encontraron que $\mathrm{K}>\mathrm{Mg}>\mathrm{Ca}$ $>\mathrm{N}>\mathrm{S}$. Las concentraciones de macronutrimentos fueron menores a las reportadas por lqbal et al. (2015), quienes en biocarbón de residuos de poda forestal reportaron para $\mathrm{N}$ 13,000, P 8000, K 7000, Ca 20,000 y Mg $6000 \mathrm{mg} \mathrm{kg}^{-1}$.

Los valores obtenidos para $\mathrm{N}$ y Ca son aproximados a los reportados por George et al. (2017), quienes encontraron 3760 y $8640 \mathrm{mg} \mathrm{kg}^{-1}$, respectivamente; pero los valores para $\mathrm{P}, \mathrm{K}$ y $\mathrm{Mg}$ son menores a los reportados por los mismos autores, de 8409, 40,395 y 10,110 mg kg-1, respectivamente.

\section{Concentración de micronutrimentos}

En lo que respecta a la concentración de micronutrientes hubo diferencias $(P \leq 0.05)$ por efecto del catalizador utilizado (Cuadro 2). Con excepción del Fe, el ácido propiónico 10 \% favoreció la concentración de los micronutrimentos. De igual manera, la concentración de micronutrimentos se favoreció con al menos una concentración de los ácidos aplicados como catalizadores, con excepción de la concentración de Cu y Na. Centurion et al. (2017) también reportaron diferencias en la concentración de micronutrimentos ( $\mathrm{Fe}, \mathrm{B}, \mathrm{Zn}, \mathrm{Cu}$ y Mn) al adicionar los ácidos sulfúrico, fosfórico y bórico a concentración de $0.1 \mathrm{M}$ en la elaboración de biocarbón a partir de mezclas de vinaza y bagazo de caña de azúcar.

Cuadro 2. Concentración de micronutrimentos y del elemento benéfico $\mathrm{Na}$ en el biocarbón de cascarilla de arroz con diferentes catalizadores.

\begin{tabular}{lcccccc}
\hline \multirow{2}{*}{ Tratamiento } & $\mathrm{Fe}$ & $\mathrm{Cu}$ & $\mathrm{Mn}$ & $\mathrm{Zn}$ & $\mathrm{Si}$ & $\mathrm{Na}$ \\
\cline { 2 - 7 } & \multicolumn{7}{c}{$\left(\mathrm{mg} \mathrm{kg}^{-1}\right)$} \\
\hline Agua (Testigo) & $77.1 \mathrm{f}$ & $53.0 \mathrm{a}$ & $75.4 \mathrm{~g}$ & $20.1 \mathrm{~b}$ & $26.3 \mathrm{bc}$ & $132.4 \mathrm{a}$ \\
Ácido maleico 5\% & $123.1 \mathrm{e}$ & $44.7 \mathrm{ab}$ & $118.0 \mathrm{e}$ & $24.8 \mathrm{~b}$ & $27.6 \mathrm{abc}$ & $130.5 \mathrm{a}$ \\
Ácido maleico 10\% & $215.2 \mathrm{c}$ & $47.7 \mathrm{ab}$ & $120.9 \mathrm{~d}$ & $25.9 \mathrm{~b}$ & $25.6 \mathrm{bc}$ & $92.8 \mathrm{~b}$ \\
Ácido cítrico 5\% & $77.1 \mathrm{f}$ & $38.6 \mathrm{~b}$ & $101.0 \mathrm{f}$ & $23.2 \mathrm{~b}$ & $30.4 \mathrm{ab}$ & $144.4 \mathrm{a}$ \\
Ácido cítrico 10\% & $230.6 \mathrm{~b}$ & $45.0 \mathrm{ab}$ & $123.7 \mathrm{c}$ & $16.7 \mathrm{~b}$ & $23.9 \mathrm{c}$ & $154.3 \mathrm{a}$ \\
Ácido propiónico 5\% & $238.3 \mathrm{a}$ & $47.7 \mathrm{ab}$ & $152.1 \mathrm{~b}$ & $22.2 \mathrm{~b}$ & $32.4 \mathrm{a}$ & $160.0 \mathrm{a}$ \\
Ácido propiónico 10\% & $199.9 \mathrm{~d}$ & $43.7 \mathrm{ab}$ & $281.4 \mathrm{a}$ & $58.4 \mathrm{a}$ & $28.7 \mathrm{abc}$ & $144.4 \mathrm{a}$ \\
CV (\%) & 8.2 & 9.5 & 5.7 & 12.8 & 7.1 & 9.3 \\
DSH (0.05) & 6.9 & 12.5 & 2.3 & 9.9 & 5.7 & 36.3 \\
\hline
\end{tabular}

Medias con letras iguales dentro de las columnas no son estadísticamente diferentes (Tukey, P $\leq 0.05)$, CV: coeficiente de variación, DSH: diferencia significativa honesta. 
En general, independientemente del catalizador empleado, la concentración de micronutrimentos en orden decreciente fue $\mathrm{Fe}>\mathrm{Mn}>\mathrm{Cu}>\mathrm{Si}>\mathrm{Zn}$ (Cuadro 2). Esta secuencia de concentración coincide parcialmente con lo reportado por George et al. (2017), quienes en biocarbón de maíz ensilado encontraron la secuencia $\mathrm{Fe}>\mathrm{Mn}>\mathrm{Zn}>$ Cu. La concentración de Cu del presente estudio fue similar a la reportada por lqbal et al. (2015), quienes en biocarbón de residuos de poda forestal encontraron $45 \mathrm{mg} \mathrm{kg}^{-1}$ de este micronutrimento, pero los valores de $\mathrm{Zn}$ y $\mathrm{Mn}$ fueron menores a los obtenidos por esos autores (183 y $464 \mathrm{mg}$ $\mathrm{kg}^{-1}$, respectivamente). Por otro lado, con excepción del $\mathrm{Cu}$, los valores del presente estudio para $\mathrm{Fe}, \mathrm{Mn}, \mathrm{Na}$, Si y $\mathrm{Zn}$ fueron menores a los reportados por George et al., (2017), quienes en biocarbón de maíz ensilado encontraron concentraciones (en $\mathrm{mg} \mathrm{kg}^{-1}$ ) de Fe 42,141, Mn 646, $\mathrm{Na}$ 3897, Zn 281 y Cu < 0.1. La concentración de Zn en el presente estudio fue mayor a la reportada por Kalderis et al. (2014), quienes en cascarilla de arroz reportaron $0.8 \mathrm{mg}$ $\mathrm{kg}^{-1}$. Estos mismos autores no detectaron la presencia de $\mathrm{Cu}$, ni cuantificaron los demás micronutrimentos.

La importancia de los resultados de la presente investigación reside en que éstos constituyen el primer reporte de la caracterización nutrimental de biocarbón de cascarilla de arroz con fines de aplicación agrícola. No se encontraron reportes en la literatura sobre concentración de micronutrimentos en función de los ácidos evaluados como catalizadores. La aplicación de los ácidos orgánicos cítrico, maleico y propiónico favorecen la concentración nutrimental de algunos macro y micronutrimentos.

Al igual que el rendimiento y el contenido de materia orgánica, la concentración de macro y micronutrimentos es variable en función del material vegetal empleado en la elaboración del biocarbón, tal como lo reportaron Kambo y Dutta (2015). En este sentido, es necesario hacer más investigaciones relacionadas con la concentración nutrimental de biocarbones con fines de mejoramiento de suelos y como fuente de nutrimentos; asimismo, es necesario explorar otros materiales vegetales disponibles localmente (Medina y Medina, 2018).

\section{Estructura del biocarbón}

Se observaron microesferas de carbón de diversos tamaños, según el catalizador empleado; los valores de diámetro promedio fueron: testigo (agua) $0.055 \mu \mathrm{m}$, ácido maleico (5 \%) $0.064 \mu \mathrm{m}$, ácido maleico (10\%) $0.082 \mu \mathrm{m}$, ácido cítrico (5\%) $0.086 \mu \mathrm{m}$, ácido cítrico (10\%) $0.084 \mu \mathrm{m}$, ácido propiónico (5\%) $0.059 \mu \mathrm{m}$ y ácido propiónico (10\%) $0.082 \mu \mathrm{m}$ (Figura 1). Una ampliación de las microesferas formadas se puede observar en la Figura 2, para el caso de ácido cítrico y ácido maleico al 5 \%.

Con base en lo anterior, se observó que con los tres ácidos aplicados como catalizadores se incrementó el tamaño de las microesferas en comparación con el testigo. Los biocarbones que se obtienen por medio de carbonización hidrotérmica (HTC) generalmente son de superficie porosa ligera y con mejor capacidad de adsorción en comparación con el biocarbón elaborado por medio de pirolisis convencional (Regmi et al., 2012). Asimismo, se ha reportado que el biocarbón producido con HTC en pH ácido incrementa 2.7 veces la superficie de área $\left(\mathrm{m}^{2} \mathrm{~g}^{-1}\right)$, el volumen de los poros $\left(1.1 \times 10^{-1} \mathrm{~mL} \mathrm{~g}^{-1}\right)$ y el tamaño de los poros $(20.2 \mathrm{~nm})$ en comparación con los producidos en pH alcalino (Reza et al., 2015), lo que podría explicar el mayor tamaño de los microesferas con la adición de los ácidos maleico, cítrico y propiónico. Adicionalmente, cabe señalar que la formación de microesferas se favorece con la técnica de HTC. En este sentido, Hu et al. (2008) no observaron microesferas en la estructura superficial de biocarbón de cascarilla de arroz elaborado con la técnica de pirolisis rápida.

El biocarbón obtenido de la cascarilla de arroz por medio de carbonización hidrotérmica y elaborado con la adición de ácidos como catalizadores se podría utilizar como mejorador de suelos agrícolas, ya que la porosidad y la presencia de las microesferas de carbón en la estructura del mismo, podrían actuar como superficie de adsorción de nutrimentos, lo cual reduce la lixiviación de minerales y podría hacer más eficiente el uso de fertilizantes a lo largo del ciclo de los cultivos, tal como lo reportaron Guiotoku et al. (2012). Si bien los resultados del presente estudio son promisorios, es necesario realizar investigaciones agronómicas para evaluar el efecto del biocarbón de cascarilla de arroz sobre el crecimiento y rendimiento de cultivos, así como su efecto en las propiedades físicoquímicas en suelos $u$ otros sustratos.

\section{CONCLUSIONES}

La adición de los los ácidos maleico y cítrico al $10 \%$ como catalizadores propician mayor rendimiento de biocarbón de cascarilla de arroz. La mayor concentración de materia orgánica se obtuvo con ácido maleico $5 \%$. En general, los tres macronutrimentos con mayor concentración fueron $\mathrm{Ca}, \mathrm{N}$ y $\mathrm{K}$, mientras que para micronutrimentos los de mayor concentración fueron Fe y Mn, así como el Na. Por sus características de rendimiento y concentración de materia orgánica y nutrimental, el biocarbón de cascarilla de arroz potencialmente se podría usar como mejorador de suelos agrícolas. 

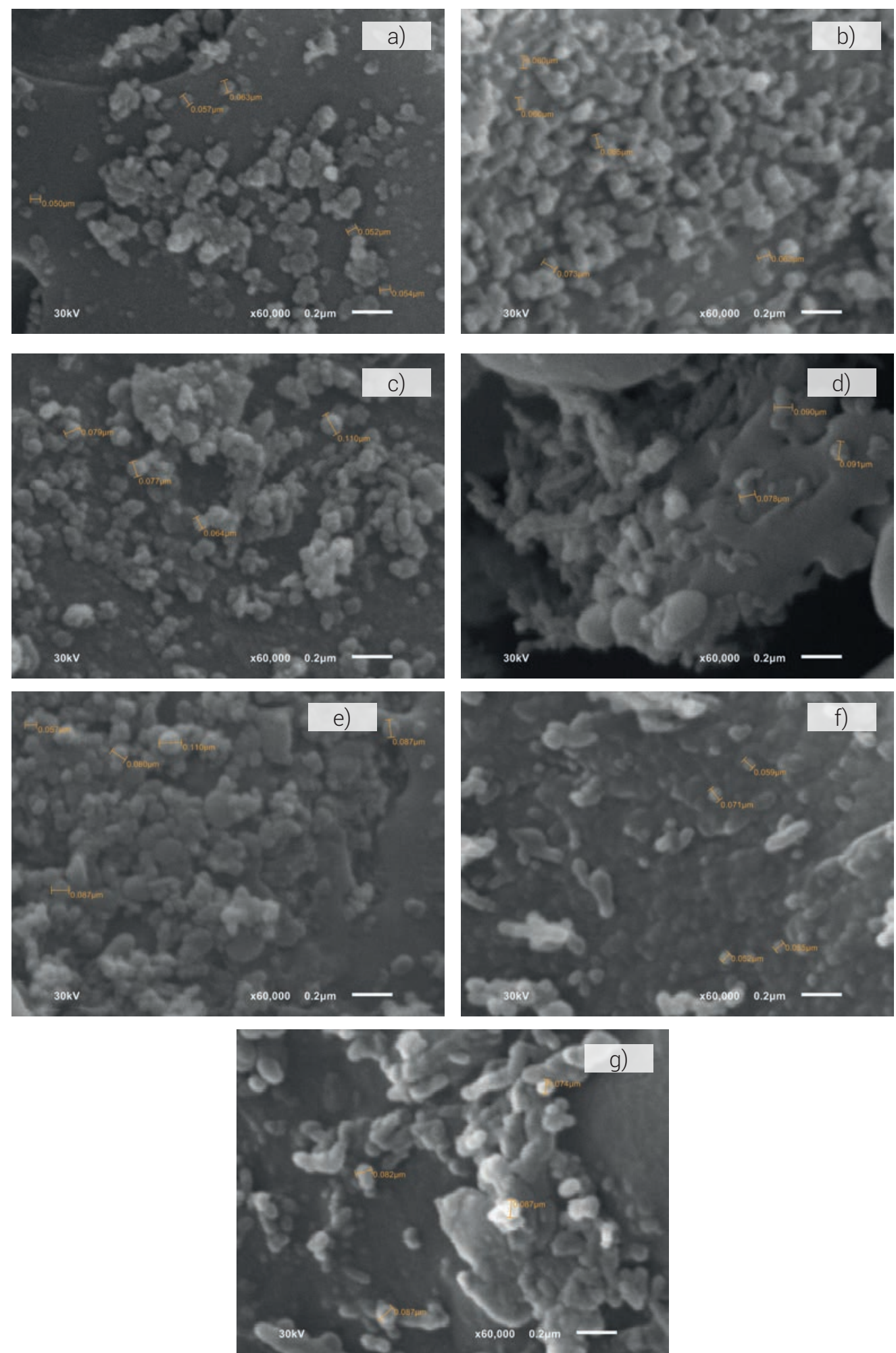

Figura 1. Microesferas en el biocarbón de cascarilla de arroz producido con diferentes catalizadores. a) agua, b) ácido maleico $5 \%$, c) ácido maleico $10 \%$, d) ácido cítrico $5 \%$, e) ácido cítrico $10 \%$, f) ácido propiónico $5 \%$ y g) ácido propiónico $10 \%$. 

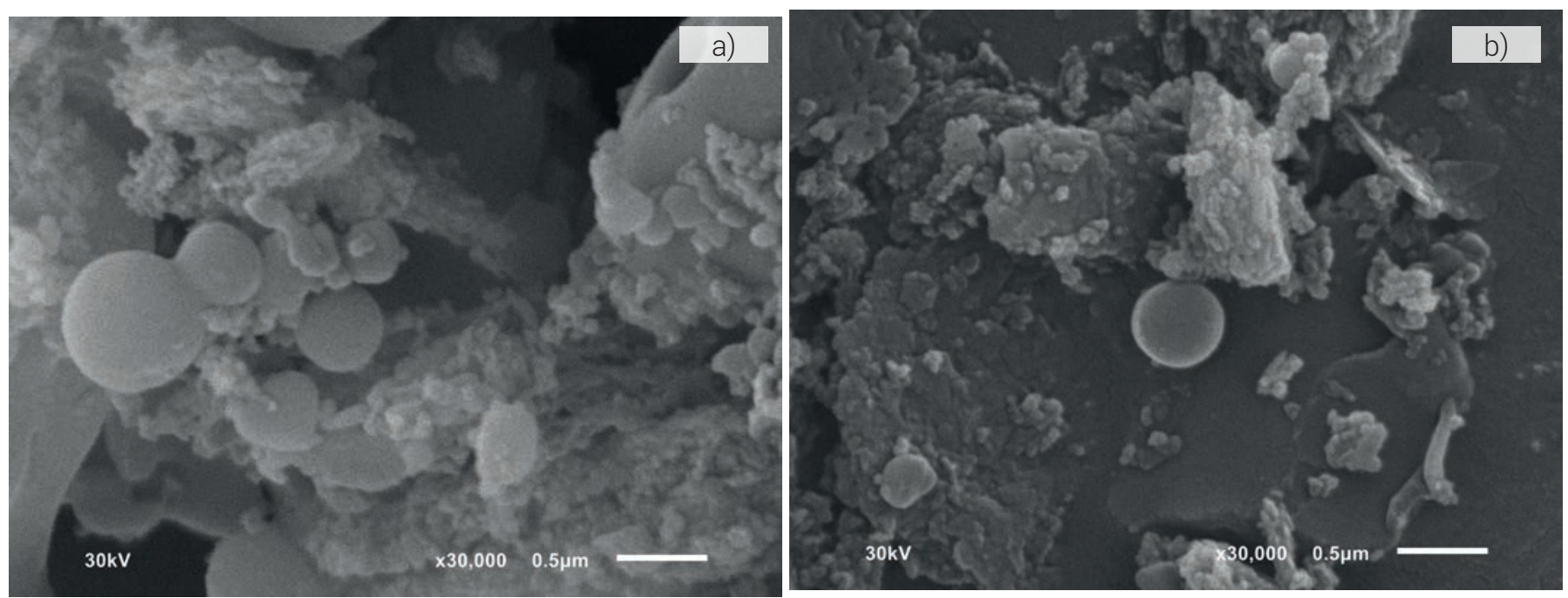

Figura 2. Microesferas en la estructura superficial de biocarbón de cascarilla de arroz elaborado con dos catalizadores: a) ácido cítrico a $5 \%$ y b) ácido maleico a $5 \%$.

\section{AGRADECIMIENTOS}

La primera autora (JVM) agradece al Consejo Nacional de Ciencia y Tecnología (CONACYT-México) por la beca otorgada para estudios de Maestría en Ciencias Agropecuarias y Desarrollo Rural.

\section{BIBLIOGRAFÍA}

Adeyemi T. O. A. and O. D. Idowu (2017) Biochar: promoting crop yield, improving soil fertility, mitigating climate change and restoring polluted soils. World News of Natural Sciences 8:27-36.

Alburquerque J. A., J. M. Calero, V. Barrón, J. Torrent, M. C. del Campillo, A. Gallardo and R. Villar (2014) Effects of biochars produced from different feedstocks on soil properties and sunflower growth Journal of Plant Nutrition and Soil Science 177:16-25, https:// doi.org/10.1002/jpln.201200652

Alcántar G. G. y M. Sandoval V. (1999) Manual de Análisis Químico de Tejido Vegetal. Guía de Muestreo, Preparación, Análisis e Interpretación. Sociedad Mexicana de la Ciencia del Suelo, A. C. Chapingo, México. $155 \mathrm{p}$.

Baccile N., M. Antonietti and M. M. Titirici (2010) One-step hydrothermal synthesis of nitrogen-doped nanocarbons: albumine directing the carbonization of glucose. ChemSusChem 3:246-253, https://doi.org/10.1002/cssc.200900124

Centurion S. C., C. A. Melo, F. H. Soares, A. B. Moreira, O. P. Ferreira and M. C. Bisinoti (2017) Effect of the reaction medium on the immobilization of nutrients in hydrochars obtained using sugarcane industry residues. Bioresource Technology 237:213221, https://doi.org/10.1016/j.biortech.2017.04.004

Ding Y., Y. Liu, S. Liu, Z. Li, X. Tan, X. Huang, G. Zeng, L. Zhou and B. Zheng (2016) Biochar to improve soil fertility. A review. Agronomy for Sustainable Development 36:36, https://doi.org/10.1007/ s13593-016-0372-z

Escalante R. A., G. Pérez L., C. Hidalgo M., J. López C., J. Campo A., E. Valtierra P. y J. D. Etchevers B. (2016) Biocarbón (biochar) I: naturaleza historia, fabricación y uso en el suelo. Terra Latinoamericana 34:367-382

George E., M. Ventura, P. Panzacchi, F. Scandellari and G. Tonon (2017) Can hydrochar and pyrochar affect nitrogen uptake and biomass allocation in poplars? Journal of Plant Nutrition and Soil Science 180:178-186, https://doi.org/10.1002/jpln.201600563 Guiotoku M., F. A. Hansel, E. H. Novotny and C. M. B. de Freitas Maia (2012)
Molecular and morphological characterization of hydrochar produced by microwave-assisted hydrothermal carbonization of cellulose. Pesquisa Agropecuária Brasileira 47:687-692, https://doi.org/10.1590/S0100-204X2012000500008

Gurwick N. P., L. A. Moore, C. Kelly and P. Elias (2013) A systematic review of biochar research, with a focus on its stability in situ and its promise as a climate mitigation strategy. PLoS ONE 8:e75932, https://doi.org/10.1371/journal.pone.0075932

Hu S., J. Xiang, L. Sun, M. Xu, J. Qiu and P. Fu (2008) Characterization of char from rapid pyrolysis of rice husk. Fuel Processing Technology 89:1096-1105, https://doi.org/10.1016/j.fuproc.2008.05.001

Ibarrola R., B. Evar y D. Reay (2013) Comercialización de Biocarbón (Biochar) en México. Definición del Contexto para un Programa de Investigación Multidisciplinario. Universidad de Edimburgo. Edimburgo, Escocia. 24 p

Iqbal H., M. Garcia-Perez and M. Flury (2015) Effect of biochar on leaching of organic carbon, nitrogen, and phosphorus from compost in bioretention systems. Science of the Total Environment 521522: 37-45, https://doi.org/10.1016/j.scitotenv.2015.03.060

Kalderis D., M. S. Kotti, A. Méndez and G. Gascó (2014) Characterization of hydrochars produced by hydrothermal carbonization of rice husk. Solid Earth 5:477-483, https://doi.org/10.5194/se-5477-2014

Kambo H. S. and A. Dutta (2015) A comparative review of biochar and hydrochar in terms of production, physico-chemical properties and applications. Renewable and Sustainable Energy Reviews 45:359-378, https://doi.org/10.1016/j.rser.2015.01.050

Lynam J. G., C. J. Coronella, W. Yan, M. T. Reza and V. R. Vasquez (2011) Acetic acid and lithium chloride effects on hydrothermal carbonization of lignocellulosic biomass. Bioresource Technology 102:6192 6199, https://doi.org/10.1016/j.biortech.2011.02.035

Lynam J. G., M. T. Reza, V. R. Vasquez and C. J. Coronella (2012) Effect of salt addition on hydrothermal carbonization of lignocellulosic biomass. Fuel 99:271-273, https://doi.org/10.1016/j. fuel.2012.04.035

Medina 0. L. E. e I. N. Medina 0. (2018) Prototipo autotérmico móvil para producción de biocarbón con biomasa de esquilmos de aquacate. Terra Latinoamericana 36:121-129, https://doi. org/10.28940/terra.v36i2.217

Onay 0̈. (2014) Effects of catalyst on pyrolysis of laurel (Laurus nobilis L.) seed in a fixed bed tubular reactor. Chemical Engineering Transactions 37:127-132, https://doi.org/10.3303/ CET1437022

Quesada-Kimsey J. (2012) La carbonización de residuos biomásicos: una exploración con perspectivas emocionantes. Revista Tecnología en Marcha 25:14-21, https://doi.org/10.18845/ tm.v25i5.465 
Regmi P., J. L. Garcia M., S. Kumar, X. Cao, J. Mao and G. Schafran (2012) Removal of copper and cadmium from aqueous solution using switchgrass biochar produced via hydrothermal carbonization process. Journal of Environmental Management 109:61-69, https://doi.org/10.1016/j.jenvman.2012.04.047

Reza M. T., E. Rottler, L. Herklotz and B. Wirth (2015) Hydrothermal carbonization (HTC) of wheat straw: influence of feedwater $\mathrm{pH}$ prepared by acetic acid and potassium hydroxide. Bioresource Technology 182:336-344, https://doi.org/10.1016/j. biortech.2015.02.024

Rodríguez A. D. y R. Grant A. (2015) La cáscara de arroz, un problema medioambiental para la ciudad de Manzanillo. Propuestas de soluciones para el desarrollo local. Revista Eco Solar 54:1-5.

Rosenani A. B., S. H. Ahmad, S. Nurul Adila and T. Wei Loon (2014) Biochar as a soil amendment to improve crop yield and soil carbon sequestration. Acta Horticulturae 1018:203-209, https://doi. org/10.17660/ActaHortic.2014.1018.20

SAS Institute (2004) SAS/STAT User's Guide. Release 9.1. SAS Institute. Cary, North Carolina, USA. 5121 p.

Schneider D., M. Escala, K. Supawittayayothin and N. Tippayawong (2011) Characterization of biochar from hydrothermal carbonization of bamboo. International Journal of Energy and Environment 2:647-652.

Schulz H., G. Dunst and B. Glaser (2013) Positive effects of composted biochar on plant growth and soil fertility. Agronomy for Sustainable Development 33:817-827, https://doi.org/10.1007/ s13593-013-0150-0

SIAP, Servicio de Información Agroalimentaria y Pesquera (2019) Anuario estadístico de la producción agrícola. Servicio de Información Agroalimentaria y Pesquera. SADER. Ciudad de México. https:// nube.siap.gob.mx/cierreagricola/ (Marzo 2019).

Varela M. O., E. B. Rivera, W. J. Huang, C. C. Chien and Y. M. Wang (2013) Agronomic properties and characterization of rice husk and wood biochars and their effect on the growth of water spinach in a field test. Journal of Soil Science and Plant Nutrition 13:251266, https://doi.org/10.4067/S0718-95162013005000022

Villamagua M., L. Valarezo, C. Valarezo, C. Tandazo C. y M. Ramón (2014) Efecto de granulometrías de biocarbón sobre las condiciones físicas del suelo y tensiones de humedad en el cultivo del tomate Solanum lycopersicum, bajo invernadero. Revista CEDAMAZ 3:93-105.

Wang S. and C. N. Mulligan (2009) Effect of natural organic matter on arsenic mobilization from mine tailings. Journal of Hazardous Materials 168:721-726, https://doi.org/10.1016/j. jhazmat.2009.02.088

Zheng W., N. Holm and K. A. Spokas (2016) Research and application of biochar in North America. In: Agricultural and Environmental Applications of Biochar: Advances and Barriers. M. Guo, Z. Heand S. M. Uchimiya (eds.). SSSA Special Publication 63. Soil Science Society of America. Madison, Wisconsin, USA. pp:475-494, https://doi.org/10.2136/sssaspecpub63.2014.0053 